International Journal of Pure and Applied Mathematics

Volume 105 No. 4 2015, 775-793

ISSN: 1311-8080 (printed version); ISSN: 1314-3395 (on-line version)

url: http://www.ijpam.eu

doi: http://dx.doi.org/10.12732/ijpam.v105i4.17

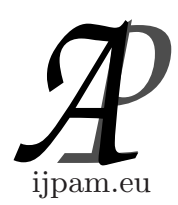

\title{
ENFORCED HAMILTONIAN CYCLES \\ IN DISTANCE GRAPHS
}

\author{
Mária Timková \\ Institute of Mathematics \\ Faculty of Sciences \\ P. J. Šafárik University \\ Jesenná 5, 04154 Košice, SLOVAKIA
}

\begin{abstract}
The H-force number of a hamiltonian graph $G$ is the smallest number $k$ with the property that there exists a set $W \subseteq V(G),|W|=k$, such that each cycle passing through all vertices of $W$ is hamiltonian. In this paper, we determine the $\mathrm{H}$-force numbers of distance graphs with two parameters.
\end{abstract}

AMS Subject Classification: 05C45

Key Words: hamiltonian graph, H-force number, distance graph, long cycle

\section{Introduction}

Throughout this paper we consider graphs without loops or multiple edges; for terminology not defined here, we refer to [3].

In research of hamiltonian graphs, there are several concepts setting a kind of stratification within this family of graphs, like the number of different cycle lengths (and the related notion of pancyclicity), the number of edges that can be prescribed in certain way such that it is possible to route a hamiltonian cycle through them (see [11], [7] or [5] for the case of 4-connected planar graphs) Another way of classification of hamiltonian graphs involves the notion of $k$ -

Received: October 7, 2015

(c) 2015 Academic Publications, Ltd. url: www.acadpubl.eu 
hamiltonicity: an $n$-vertex graph $G=(V, E)$ is called $k$-hamiltonian if, for all sets $U \subseteq V, 0 \leq|U| \leq k$, the graph $G-U$ (obtained from $G$ by deleting all vertices of $U$ ) is hamiltonian. In particular, a graph is 1-hamiltonian if it is hamiltonian and the graph that results from deletion of any vertex is also hamiltonian. There are several sufficient conditions for graphs to be 1hamiltonian (see [1], [2] or [10]); in many cases, these conditions are similar to the classical conditions for hamiltonian connectedness.

Yet another concept of developing a hierarchy within hamiltonian graphs was defined first in [4] in the following way: let $G=(V, E)$ be a hamiltonian graph and let $W \subseteq V, W \neq \emptyset$. A cycle in $G$ is a $W$-cycle if it contains all vertices of $W$. We say that $W$ enforces a hamiltonian cycle in $G$ (or, $W$ is an $H$-force set) if each $W$-cycle of $G$ is hamiltonian. The $H$-force number $h(G)$ is the cardinality of a smallest $\mathrm{H}$-force set in $G$.

Note that if a graph $G=(V, E)$ is 1-hamiltonian, then $h(G)=|V|$, and vice versa. Thus, it is natural to consider the graphs with $\mathrm{H}$-force number being less than their orders. The graphs with small H-force number were studied in [4], where was given, among other results, the complete characterization of graphs with $\mathrm{H}$-force number 2 (or 3 in the case of 3-connected graphs, and 4 for 3-connected planar graphs, respectively).

In general, determining the H-force number of a hamiltonian graph is a difficult problem, even for special graphs. In the paper [12], there were determined H-force numbers of generalized dodecahedra (that is, 3-connected planar cubic graphs consisting of two $k$-gonal faces separated by the strip of $2 k$ pentagons). In this paper, we deal with $\mathrm{H}$-force numbers of distance graphs, defined as follows: for a finite set of positive integers $D \subseteq \mathbb{N}$, the distance graph $G_{n}(D)$ has vertex set $V\left(G_{n}(D)\right)=[0, n-1]=\{0,1, \ldots, n-1\}$; two vertices $u$ and $v$ of $G_{n}(D)$ are adjacent if $|u-v| \in D$. Here, we consider the case $D=\left\{d_{1}, d_{2}\right\}$ (using the notation $G_{n}\left(d_{1}, d_{2}\right)$ for the corresponding distance graph). The hamiltonian properties of $G_{n}\left(d_{1}, d_{2}\right)$ were studied in [9]; it was proved that, for sufficiently large $n$ and for coprime indivisible integers $d_{1}, d_{2}$, the graph $G_{n}\left(d_{1}, d_{2}\right)$ is always hamiltonian (further, in [8] it was shown that such a graph contains hamiltonian path connecting vertices 0 and $n-1)$. In addition to these results, we determine the exact values of $\mathrm{H}$-force numbers of graphs $G_{n}\left(d_{1}, d_{2}\right)$ with $\frac{d_{2}}{d_{1}} \geq 2$ and an upper bound in the case $\frac{d_{2}}{d_{1}}<2$. 


\section{The Structure of $G_{n}\left(d_{1}, d_{2}\right)$}

In this section, we describe a special representation of the graph $G_{n}\left(d_{1}, d_{2}\right)$ featuring the existence of its particular grid-like subgraph.

Let $d_{1}$ and $d_{2}$ be positive integers with $\operatorname{gcd}\left(d_{1}, d_{2}\right)=1, d_{1}<d_{2}$ and let $X=$ $\left[0, d_{2}-1\right]$ and $Y=\left[-d_{1}+1, \infty\right)$. Given a vertex $v \in[0, n-1]$ of $G_{n}\left(d_{1}, d_{2}\right)$, there exist unique integers $x \in X$ and $y \in Y$ such that $v=x d_{1}+y d_{2}$. The ordered pair $(x, y)$ thus forms the coordinates of $v$ in $G_{n}\left(d_{1}, d_{2}\right)$. In the following, we will use the notation $(x, y) \in V\left(G_{n}\left(d_{1}, d_{2}\right)\right)$ meaning that $v$ with coordinates $(x, y)$ is a vertex of $G_{n}\left(d_{1}, d_{2}\right)$, and we will use the addition/subtraction $(x, y) \pm k=\left(x^{\prime}, y^{\prime}\right)$ to express the fact that, for two vertices $v, v^{\prime} \in[0, n-1]$ with coordinates $(x, y)$ and $\left(x^{\prime}, y^{\prime}\right), v \pm k=v^{\prime}$ holds (in a similar manner, we will use the comparison $(x, y) \leq k)$. In this way, for a vertex $(x, y)$ of $G_{n}\left(d_{1}, d_{2}\right)$, we have

$$
\begin{aligned}
(x, y)+d_{1} & =(x+1, y) \text { if } x<d_{2}-1 \\
(x, y)-d_{1} & =(x-1, y) \text { if } x>0 \\
\left(d_{2}-1, y\right)+d_{1} & =\left(0, y+d_{1}\right) \text { if }\left(d_{2}-1, y\right) \in\left[0, n-d_{1}-1\right] \\
(0, y)-d_{1} & =\left(d_{2}-1, y-d_{1}\right) \text { if }(x, y) \in\left[d_{1}, n-1\right] \\
(x, y)+d_{2} & =(x, y+1) \text { if }(x, y) \notin\left[0, n-d_{2}-1\right] \\
(x, y)-d_{2} & =(x, y-1) \text { if }(x, y) \notin\left[d_{2}, n-1\right]
\end{aligned}
$$

which implies that the vertex $(x, y)$ is adjacent to the vertex $(x+1, y)$ (or $\left.\left(0, y+d_{1}\right)\right),(x-1, y)$ (or $\left.\left(d_{2}-1, y-d_{1}\right)\right),(x, y-1)$ if $(x, y-1) \in V(G)$, and $(x, y+1)$ if $(x, y+1) \in V(G)$ (see Fig. 1). In the sequel, we will identify the vertices written in coordinate form with vertices of the lattice $\mathbb{Z}^{2}$ or vertices of an unit square mesh on cylinder.

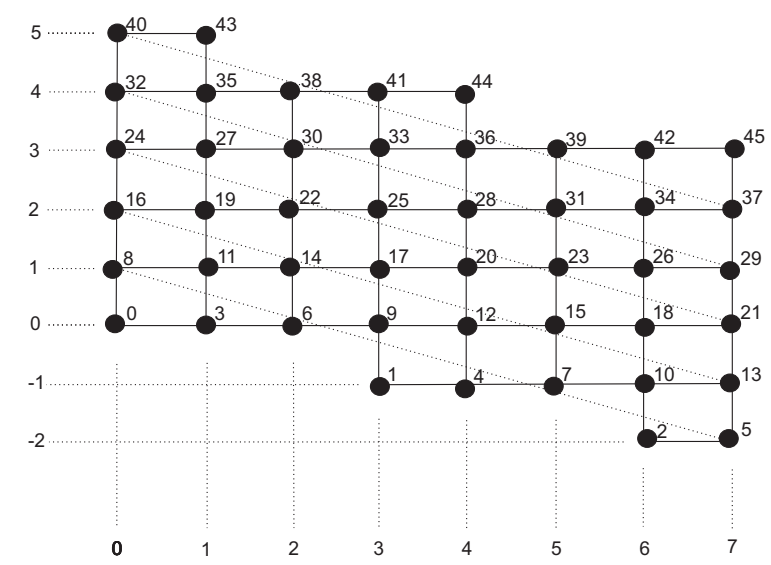


Fig. 1: The representation of $G_{46}(3,8)$

In the grid representation of $G_{n}\left(d_{1}, d_{2}\right)$, all vertices $(x, y)$ with $x=c$ form the column $c$; similarly, all vertices $(x, y)$ satisfying $y=r$ form the row $r$. For an integer $c$, let $u(c)=\max \left\{y:(c, y) \in V\left(G_{n}\left(d_{1}, d_{2}\right)\right)\right\}$ and $l(c)=\min \left\{y:(c, y) \in V\left(G_{n}\left(d_{1}, d_{2}\right)\right)\right\}$. The columns $x, x+1, \ldots, x+w-1$ form a block of width $w$ (a $w$-block), if the vertices $(x, l(x)),(x+w, l(x+w))$ are 2 -vertices and none of vertices $(x+i, l(x+i)), i \in[1, w-1]$ is a 2-vertex. Let $B_{1}, B_{2}, \ldots, B_{k}$ be the consecutive blocks of distance graph $G=G_{n}\left(d_{1}, d_{2}\right)$; the block $B_{1}$ is the first block, the block $B_{k}$ is the terminal block, and the characteristic $\left(w_{1}, w_{2}, \ldots, w_{k}\right)$ of $G$ is an ordered $k$-tuple where $w_{i}$ is the width of the block $B_{i}, i \in\{1,2, \ldots, k\}$.

Lemma 1. Let $G=G_{n}\left(d_{1}, d_{2}\right)$ be a distance graph. Then

1. the width of any block of $G$ is either $\left\lfloor\frac{d_{2}}{d_{1}}\right\rfloor$ or $\left\lceil\frac{d_{2}}{d_{1}}\right\rceil$;

2. G has exactly $d_{1}$ blocks.

3. the width of the first block is $\left\lceil\frac{d_{2}}{d_{1}}\right\rceil$ and the width of terminal block is $\left\lfloor\frac{d_{2}}{d_{1}}\right\rfloor ;$

Proof. 1. Let $x, x+1, \ldots, x+w-1$ be the columns of the $w$-block $B$ and $y=l(x)$. The vertex $(x, y)$ is a 2 -vertex, so $(x, y) \leq d_{1}-1$. The vertex $(x+1, y)$ is a neighbour of a 2 -vertex, so $d_{1} \leq(x+1, y)$, similarly, the vertex $(x+w, y)$ is a neighbor of a 2 -vertex, so $d_{2} \leq(x+w, y) \leq d_{1}+d_{2}-1$. Hence, for all integers $y$ and $k$ with $x \leq k \leq x+w-1$, we have $(k+1, y)-(k, y)=d_{1}$, therefore $(x+w, y)-(x, y)=w \cdot d_{1}$, which implies the claim.

3. $G$ has $d_{1} 2$-vertices $(x, l(x))$ and thus the same number of blocks.

2. From the first part of the proof, we obtain $\left(\left\lceil\frac{d_{2}}{d_{1}}\right\rceil-1\right) d_{1}<d_{2}$, therefore the block $B_{1}$ is a $\left\lceil\frac{d_{2}}{d_{1}}\right\rceil$-block. Let $(x, l(x))$ be a 2-vertex of $B_{d_{1}}$, then $(x, l(x))<d_{1}$ and $(x+w-1, l(x))+d_{1}=\left(0, l(x)+d_{1}\right)=d_{2}$. Hence $B_{d_{1}}$ is a $\left\lfloor\frac{d_{2}}{d_{1}}\right\rfloor$-block.

Note, that $G_{n}\left(d_{1}, d_{2}\right)$ has an automorphism mapping any vertex $u \in$ $V\left(G_{n}(D)\right)$ to the vertex $v$, where $n-u-1 \equiv v(\bmod n)$.

Several results of the next section are formulated for wider family $\mathcal{H}$ of special hamiltonian graphs, which is defined as follows: let $h \geq 4$ be an even number and let $\left(l_{1}, \ldots, l_{k}\right)$ be a sequence of integers with $l_{i} \geq 2$, for each $i \in\{1, \ldots, k\}$. Let $M_{i}, i \in\{1, \ldots, k\}$, be rectangular grid of width $l_{i}$ and 
height $h$ (i.e. $M_{i}$ is the Cartesian product of paths on $l_{i}+1$ and $h+1$ vertices, respectively) embedded in the lattice $\mathbb{Z}^{2}$ such that, for each $i \in\{1, \ldots, k\}$, the lower left corner of $M_{i}$ has Cartesian coordinates $\left(\sum_{j=1}^{i-1} l_{j}, 1-i\right)$. Then the graph $H\left(h ; l_{1}, \ldots, l_{k}\right)$ is obtained from the above mentioned rectangles by adding all edges $((x, y),(x+1, y))$ where $(x, y)$ is a vertex on the right side of $M_{i}$ which is different from its upper right corner, and also by adding all edges $((x, y),(0, y+k))$ where $(x, y)$ is a vertex of $M_{k}$ on its right side. The sequence $\left(l_{1}, \ldots, l_{k}\right)$ is the characteristic of $H\left(h ; l_{1}, \ldots, l_{k}\right)$. It is easy to see that each distance graph $G_{n}\left(d_{1}, d_{2}\right)$ is a subgraph of some graph $H\left(h ; l_{1}, \ldots, l_{k}\right)$, but the converse is not true in general. Let $G=G_{n}\left(d_{1}, d_{2}\right)$ and $\left(w_{1}, w_{2}, \ldots, w_{d_{1}}\right)$ be the characteristic of $G$. Let $H=H\left(h, w_{i}, w_{i+1}, \ldots, w_{d_{1}}, w_{1}, w_{2}, \ldots, w_{i-1}\right), i \in\left[1, d_{1}\right]$ and $n=h d_{2}$. Note that $G$ has an automorphism mapping any vertex $u=$ $(x, y) \in V(G)$ to the vertex of $\left(x^{\prime}, y^{\prime}\right) \in V(H)$, where $x^{\prime}=x+\sum_{j=i}^{d_{1}} w_{j}\left(\bmod d_{2}\right)$, $y^{\prime}=y-i+1$ for $x \leq \sum_{j=0}^{i-1} w_{j}$ and $y^{\prime}=y+i-1$ for $x>\sum_{j=0}^{i-1} w_{j}$.

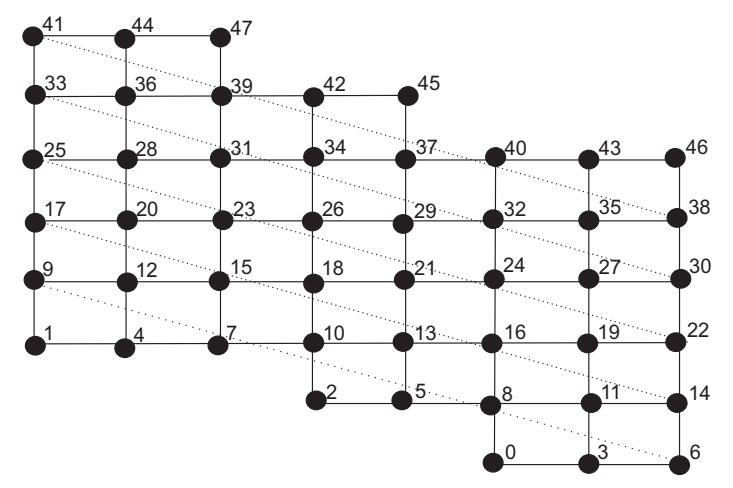

Fig. 2: The graph $H(6 ; 3,2,3) \cong G_{48}(3,8)$

\section{The Upper Bound}

Let a graph $G_{n}\left(d_{1}, d_{2}\right)$ be hamiltonian (see [9]). In this section we derive an upper bound of its H-force number. Due to the fact that $h\left(G_{n}(1,2)\right)=2$ for any $n \geq 3$ (H-force set consists of vertices 0 and $n-1$ ), we will consider only the case $\frac{d_{2}}{d_{1}} \neq 2$. For the purposes of the proof, we define an alternating path in 
$G_{n}\left(d_{1}, d_{2}\right)$ to be a path whose vertices are alternately of degree two and more than two, while the degree of its initial vertex is four and degree of its terminal vertex is three.

Lemma 2. Let $G=G_{n}\left(d_{1}, d_{2}\right)$ be a non-bipartite hamiltonian distance graph. Then $h(G) \leq n-f\left(d_{1}, d_{2}\right)$, where

$$
f\left(d_{1}, d_{2}\right)= \begin{cases}4 d_{1}, & \text { if } \frac{d_{2}}{d_{1}}>2 \text { and } n \geq 4 d_{2}, \\ 3 d_{1}, & \text { if } \frac{d_{2}}{d_{1}}<2 \text { and } n \geq 8 d_{2}, \\ \frac{1}{2} d_{1}\left(d_{1}+4\right)+2, & \text { if } d_{2}=d_{1}+1, n \geq 2\left\lceil\frac{d_{1}}{d_{2}-d_{1}}\right\rceil d_{2}, \\ \frac{1}{2}\left(d_{1}+3\right)\left(d_{1}+1\right)+2, & \text { and } d_{1} \text { even, } d_{2}=d_{1}+1, n \geq 2\left\lceil\frac{d_{1}}{d_{2}-d_{1}}\right\rceil d_{2}, \\ & \text { and } d_{1} \text { odd. }\end{cases}
$$

Proof.

1. Let $G=G_{n}\left(d_{1}, d_{2}\right)$ be a non-bipartite hamiltonian graph with $n \geq 4 d_{2}$ and $\frac{d_{2}}{d_{1}}>2$. The width of each block is at least 2. Every cycle containing a 2 -vertex must contain both its neighbours as well. Thus no neighbour of a 2 -vertex can belong to a smallest $\mathrm{H}$-force set. The set $S$ of neighbours of 2 -vertices has $4 d_{1}$ vertices (because there are $2 d_{1} 2$-vertices in $G$ ). The set $W=V(G) \backslash S$ is an H-force set in $G$ hence $h(G) \leq n-4 d_{1}, f\left(d_{1}, d_{2}\right)=4 d_{1}$.

2. Let $G=G_{n}\left(d_{1}, d_{2}\right)$ be non-bipartite hamiltonian graph with $\frac{d_{2}}{d_{1}}<2$. The width of each block is 1 or 2 . The graph $G$ has $d_{2}-d_{1}$ blocks of width 2 and $2 d_{1}-d_{2}$ blocks of width 1 (remember that the number of block is $d_{1}$ and the number of columns is $\left.d_{2}\right)$. Let $u=\left(x_{1}, y_{1}\right)$ and $v=\left(x_{2}, y_{2}\right)$ be two different 3 -vertices such that there is no 3 -vertex $(x, y)$ with $x_{1}<x<x_{2}$. Then $u, v \in\left[0+d_{1}, d_{2}-1\right]$ and $d(u, v)=2 m+1$. From the conditions $u+(m+1) d_{1}=v+m d_{2}$ we obtain $m=\frac{d_{1}}{d_{2}-d_{1}}+\frac{u-v}{d_{2}-d_{1}}$ and $\frac{u-v}{d_{2}-d_{1}} \in(-1,1)$. This implies $2\left\lfloor\frac{d_{1}}{d_{2}-d_{1}}\right\rfloor \leq 2 m \leq 2\left\lceil\frac{d_{1}}{d_{2}-d_{1}}\right\rceil$. Now we can say, that there are $2 k$ alternating paths of length $s=2\left\lfloor\frac{d_{1}}{d_{2}-d_{1}}\right\rfloor$ and $2 l$ alternating paths of length $t=2\left\lceil\frac{d_{1}}{d_{2}-d_{1}}\right\rceil$.

Note that $\frac{d_{1}}{d_{2}-d_{1}}$ is not an integer. From $k+l=d_{2}-d_{1}$ and $k\left(\left\lfloor\frac{d_{1}}{d_{2}-d_{1}}\right\rfloor\right)+$ $\left(d_{2}-d_{1}-k\right)\left(\left\lfloor\frac{d_{1}}{d_{2}-d_{1}}\right\rfloor+1\right)=d_{1}$ we obtain $k=d_{2}-2 d_{1}+\left(d_{2}-d_{1}\right)\left\lfloor\frac{d_{1}}{d_{2}-d_{1}}\right\rfloor$ and $l=d_{1}-\left(d_{2}-d_{1}\right)\left\lfloor\frac{d_{1}}{d_{2}-d_{1}}\right\rfloor$.

Obviously, $W_{0}=V(G)$ is an H-force set. Let $\frac{s}{2}$ be even; thus $\frac{t}{2}$ is odd 
(analogously for $\frac{s}{2}$ odd). As in the previous case, the neighbours of 2vertices don't belong to the smallest H-force set. Let $S_{0}$ be the set of neighbours of 2-vertices and let $W_{1}=W_{0}-S_{0}, W_{1}$ is smaller $\mathrm{H}$-force set than $W_{0}$.

$W_{1}$ constains all 2-vertices. Let $P_{i}$ be an alternating path, then there are 2-vertices in $G \backslash \bigcup P_{i}$, for every alternating path $P_{i}$. Let $S_{1}$ be the set of neighbours of these vertices. Vertices from $S_{1}$ don't belong to the smallest H-force set. The set $W_{2}=W_{1}-S_{1}$ is a smaller $\mathrm{H}$-force set than $W_{1}$. In such a way we create the sets $S_{r}$ and $W_{r}$ for $r \leq \frac{1}{2}\left\lceil\frac{d_{1}}{\left(d_{2}-d_{1}\right)}\right\rceil$.

For $1 \leq i \leq k$, let $\left(x_{i}, y_{i}\right)$ be an initial vertex of a $s$-path and let $p \in$ $\left[0, \frac{s}{2}-2 r\right]$. We define

$S_{i}=\bigcup_{r=0}^{\frac{5}{2}}\left\{\left(x_{i}+p+2 r, y_{i}-p\right), n-\left(x_{i}+p+2 r, y_{i}-p\right)-1\right\}$ and similarly, for $y_{j} \leq 0$ and $1 \leq j \leq l$, let $\left(x_{j}, y_{j}\right)$ be an initial vertex of $t$-path and let $p \in\left[0, \frac{t-1}{2}-2 r\right]$. We define

$T_{j}:=\bigcup_{r=0}^{\frac{\mathrm{t}-1}{2}}\left\{\left(x_{j}+p+2 r, y_{j}-p\right), n-\left(x_{j}+p+2 r, y_{j}-p\right)-1\right\}$.

Now $W=V(G) \backslash \bigcup_{i=1}^{k} S_{i} \backslash \bigcup_{j=1}^{l} T_{j}$ is an H-force set and $f\left(d_{1}, d_{2}\right)=\sum_{i=1}^{k}\left|S_{i}\right|+$ $\sum_{j=1}^{l}\left|T_{j}\right|=k \frac{(s+6)(s+2)}{8}+l \frac{t(t+8)}{8} \geq 3 d_{1}$.

3. Let $G=G_{n}\left(d_{1}, d_{2}\right)$ be non-bipartite hamiltonian graph with $d_{2}=d_{1}+1$. From the previous case we have, that $W$ is an H-force set of $G$.

Now the set $W^{\prime}=W \backslash\{(1,1), n-(1,1)-1\}$ is a smaller H-force set (we have two alternating paths of length $\left.2 d_{1}\right)$. Now $f\left(d_{1}, d_{2}\right)=\frac{d_{1}\left(d_{1}+4\right)}{2}+2$ for $d_{1}$ even and $f\left(d_{1}, d_{2}\right)=\frac{\left(d_{1}+3\right)\left(d_{1}+1\right)}{2}+2$ for $d_{1}$ odd.

Lemma 3. Let $G=G_{n}\left(d_{1}, d_{2}\right)$ be a bipartite hamiltonian distance graph. If $n \geq 4 d_{2}$, then $h(G) \leq \frac{n}{2}-f\left(d_{1}, d_{2}\right)$, where

$$
f\left(d_{1}, d_{2}\right)= \begin{cases}d_{1}, & \text { if } \frac{d_{2}}{d_{1}}>2 \\ d_{2}-d_{1}, & \text { if } \frac{d_{2}}{d_{1}}<2\end{cases}
$$

Proof. Let $G=G_{n}\left(d_{1}, d_{2}\right)$ be a bipartite hamiltonian graph with $n \geq 2\left(d_{1}+d_{2}\right)$. Obviously $h(G) \leq \frac{n}{2}$ (both bipartitions are H-force sets of $G$ ).

Let $W^{\prime}=\{(x, y) \in V(G),(x, y) \equiv 0(\bmod 2)\}$ and $S \subset W^{\prime}$ be a set of neighbours of 2-vertices. Let $T \subset V(G) \backslash W^{\prime}$ be a set of 2-vertices. Put 
$W=\left(W^{\prime} \backslash S\right) \cup T, W$ is an H-force set. If $\frac{d_{2}}{d_{1}}>2$, we obtain $f\left(d_{1}, d_{2}\right)=d_{1}$ and if $\frac{d_{2}}{d_{1}}<2$ (similarly as in the proof of the Lemma 2, Case 2; here we have $2(k+l)$ alternating paths) we obtain $f\left(d_{1}, d_{2}\right)=k+l=d_{2}-d_{1}$.

\section{Lower Bound for Non-Bipartite Graphs}

In this section, we establish lower bound of the H-force number for non-bipartite graphs satisfying $\frac{d_{2}}{d_{1}}>2$.

A cycle $C$ in $G_{n}\left(d_{1}, d_{2}\right)$ is special, if $V(C)=V=[\min (V), \max (V)]$. It is a simple observation that every $G_{n}\left(d_{1}, d_{2}\right)$ contains a special cycle of length $d_{1}+d_{2}([9])$. For $n \equiv 0\left(\bmod d_{2}\right)$, every subgraph induced by the vertices of a simple block of $G_{n}\left(d_{1}, d_{2}\right)$ is a grid (i.e. $\left.G_{n}\left(d_{1}, d_{2}\right) \in \mathcal{H}\right)$. A pair of edges $\left(\left(x_{1}, y_{1}\right),\left(x_{2}, y_{2}\right)\right),\left(\left(x_{1}, y_{1}+1\right),\left(x_{2}, y_{2}+1\right)\right)$ is a biline pair. A cycle $C$ of $H \in \mathcal{H}$ is suitable if it contains between any two consecutive blocks $B$ and $B^{*}$ either a biline paar of edges or exactly one edge and a cycle $C$ of $H \in \mathcal{H}$ is super suitable if it contains between any two consecutive blocks $B$ and $B^{*}$ either a biline paar of edges or exactly one edge $\left(\left(x_{1}, y_{1}\right),\left(x_{2}, y_{2}\right)\right)$ with $y_{2} \neq\left[\frac{u\left(x_{2}\right)+l\left(x_{2}\right)}{2}\right]$. A cycle of $G$ misses exactly the vertices of a set $W$ (here $|W| \leq|V(G)|-3$ ), if $C$ contains all vertices of $V(G)-W$ (i.e. $C$ is a $(V(G) \backslash W)$-cycle).

Claim 4. For a nonhamiltonian cycle $C$ of $G$, every $H$-force set of $G$ contains a vertex of $V(G) \backslash V(C)$.

First, we show that for certain particular values of $n, d_{1}>1$ and $d_{2}$, the H-force number of $G_{n}\left(d_{1}, d_{2}\right)$ is equal to $n-4 d_{1}$ :

Lemma 5. The graph $G=G_{24}(1,4)$ is hamiltonian and $h(G)=20$.

Proof. It is easy to check that $G$ is hamiltonian and there is a hamiltonian cycle with one biline pair of edges do not belonging to the only block of $G$ (first cycle on Fig.3). By Lemma 2 we have $h(G) \leq 20$. Moreover, there are 20 cycles in $G$ missing exactly one of 20 different vertices and by 4 all these 20 vertices belong to any $\mathrm{H}$-force set (due to automorphism of $G$ mapping any vertex $(x, y)$ to the vertex $n-(x, y)-1$ we present only 10 cycles on Fig. 3$)$. These cycles contain only one edge does not belonging to the block of $G$. 

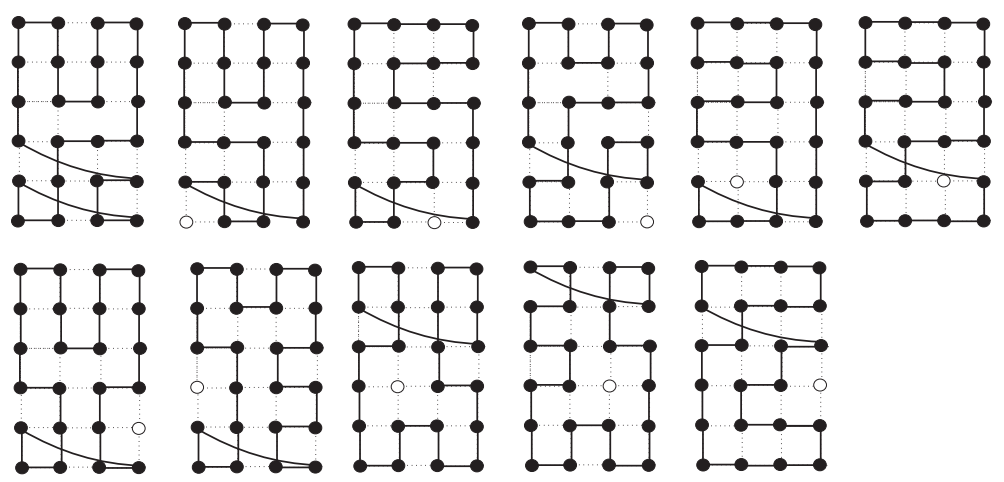

Fig. 3: Hamiltonian cycle and near-hamiltonian cycles in $G_{24}(1,4)$
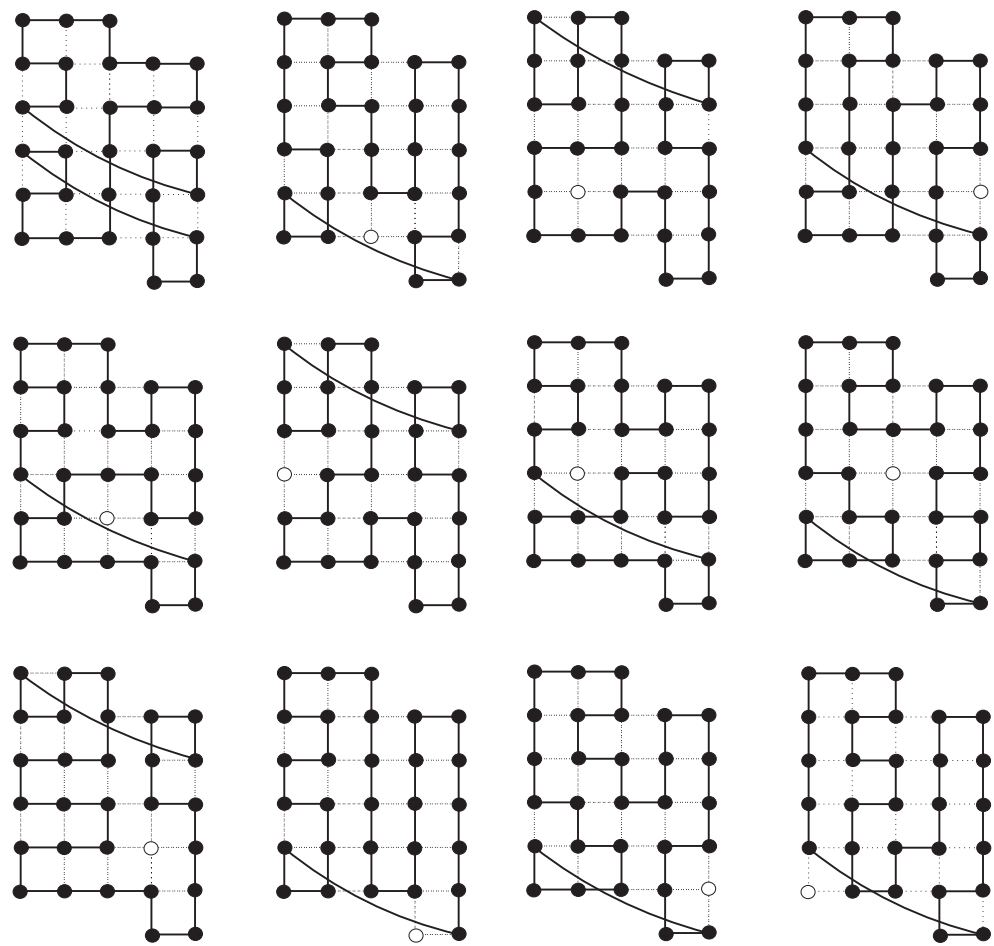

Fig. 4: Hamiltonian cycle and near-hamiltonian cycles in $G_{30}(2,5)$

Lemma 6. The graph $G=G_{30}(2,5)$ is hamiltonian and $h(G)=22$.

Proof. It is easy to check that $G$ is hamiltonian and there is a hamiltonian cycle with one biline pair of edges between every two neighboring blocks (see 
first cycle on Fig. 4). By Lemma 2 we have $h(G) \leq 22$. Moreover, there are 22 cycles in $G$ missing exactly one of 22 different vertices and by 4 all these 22 vertices belong to any $H$-force set (due to automorphism of $G$ mapping any vertex $(x, y)$ to the vertex $n-(x, y)-1$ we present only 11 of mentioned cycles on Fig. 4). These cycles are super suitable (except of cycles which miss exactly one vertex $(1,2)$ or $(2,1)$ or $(4,0)$.

Lemma 7. Let $G=G_{n}\left(d_{1}, d_{2}\right)$ be a connected distance graph with $n=$ $6 d_{2}$, let $B_{i}, B_{i+1}, 1 \leq i \leq d_{1}-2$ be two neighbouring 2-blocks of $G$, and let $(x, y)$ with $y \neq\left\lceil\frac{u(x)+l(x)}{2}\right\rceil$, be the vertex of the first column of $B_{i}$. There is a path $P_{H}^{1}$ between vertices $(x, y)$ and $(x+3, y-2)$ containing all vertices of $B_{i}$ and $B_{i+1}$ and exactly one edge between these blocks.

For the proof see Fig. 5.
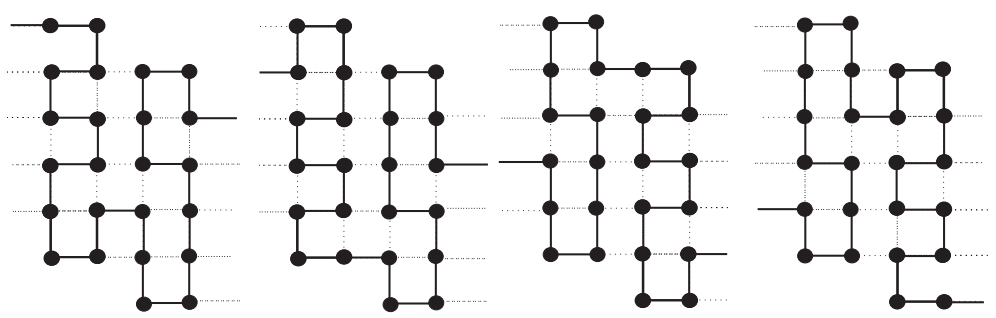

Fig. 5: Paths $P_{H}^{1}$

Lemma 8. Let $G=G_{n}\left(d_{1}, d_{2}\right)$ be a connected distance graph with $n=$ $6 d_{2}$, let $B_{i}, B_{i+1}, 1 \leq i \leq d_{1}-1$, be two neighbouring 2-blocks of $G$, and let $(x, y)$ be the vertex of the first column of $B_{i}$. There are two disjoint paths $R_{1}$ (between $(x, y)$ and $(x+3, y-2)$ ) and $R_{2}$ (between $(x, y+1)$ and $(x+3, y-1)$ ) containing (in union) all vertices of $B_{i}$ and $B_{i+1}$ and both containing exactly one edge between blocks $B_{i}, B_{i+1}$ which create together a biline par.

For the proof see Fig. 6.
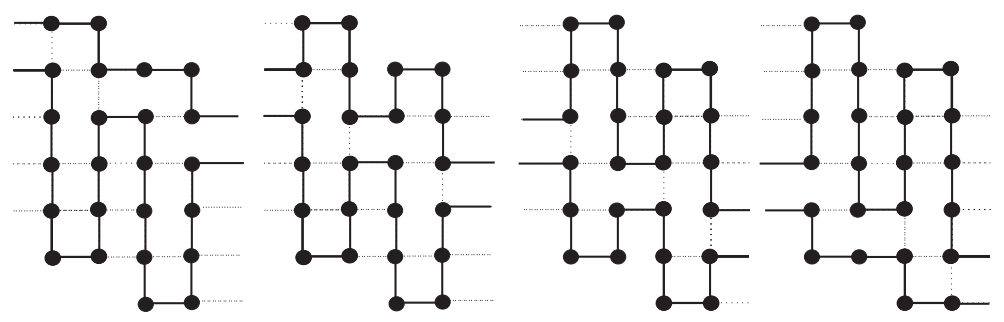

Fig. 6: Paths $R_{1}, R_{2}$ 
In the sequel, we denote the subgraph induced by edges of $R_{1}$ and $R_{2}$ as $P_{H}^{2}$.

Lemma 9. Let $G=G_{n}\left(d_{1}, d_{2}\right)$ be a connected distance graph with even $d_{1}, d_{2}=2 d_{1}+1$ and $n=6 d_{2}$. Then $G$ is hamiltonian and $h(G)=n-4 d_{1}$.

Proof. Let $G=G_{2 d_{1}+1}\left(d_{1}, 2 d_{1}+1\right)$ be a distance graph with even $d_{1}$. The characteristic of $G$ is $(3,2,2, \ldots, 2)$, with odd number of 2-blocks. By Lemma 2 we have $h(G) \leq n-4 d_{1}$; moreover, this bound is sharp for the graph $G_{30}(2,5)$. Let $G^{\prime}=G_{6\left(2 d_{1}-3\right)}\left(d_{1}-2,2 d_{1}-3\right)$.

If $d_{1} \geq 5$.

1. A hamiltonian cycle $C$ of $G$ (a cycle $C$ of $G$ which misses exactly one vertex $(x, y)$ with $\left.x \in B_{i}, i \geq d_{1}-1\right)$ will be constructed from a hamiltonian cycle $C^{\prime}$ of $G^{\prime}$ (a cycle $C^{\prime}$ of $G^{\prime}$ which misses the vertex $(x-4, y+2)$ ) and a path system $P_{H}^{2}$ (a path $P_{H}^{1}$ ) in blocks $B_{2}$ and $B_{3}$. The cycle $C$ consists of the following edges:

$\left(\left(x_{1}, y_{1}\right),\left(x_{2}, y_{2}\right)\right)$ if $\left(\left(x_{1}, y_{1}\right),\left(x_{2}, y_{2}\right)\right) \in E\left(C^{\prime}\right)$ and $x_{1} \in B_{1}$ or $x_{2} \in B_{1}$, $\left(\left(x_{1}+4, y_{1}-2\right),\left(x_{2}+4, y_{2}-2\right)\right)$ if $\left(\left(x_{1}, y_{1}\right),\left(x_{2}, y_{2}\right)\right) \in E\left(C^{\prime}\right)$ and $x_{1} \in B_{i}$ or $x_{2} \in B_{i}, i \geq 2$ and edges of a path system $P_{H}^{2}$ (a path $P_{H}^{1}$ ) in blocks $B_{2}, B_{3}$. Note that the cycle $C$ is super suitable.

2. A cycle $C$ of $G$ which misses exactly one vertex $(x, y)$ with $x \in B_{i}$, $i \leq d_{1}-2$ will be constructed from a cycle $C^{\prime}$ of $G^{\prime}$ which misses the vertex $(x, y)$ and a path $P_{H}^{1}$ in blocks $B_{d_{1}-1}, B_{d_{1}}$. The cycle $C$ consists of the following edges:

$\left(\left(x_{1}, y_{1}\right),\left(x_{2}, y_{2}\right)\right)$ if $\left(\left(2 d_{1}-4, y_{1}^{\prime}\right),\left(0, y_{1}^{\prime}+d_{1}\right)\right) \neq\left(\left(x_{1}, y_{1}\right),\left(x_{2}, y_{2}\right)\right) \in E\left(C^{\prime}\right)$ and edges $\left(\left(2 d_{1}-4, y_{1}\right),\left(2 d_{1}-3, y_{1}\right)\right), P_{H}^{1},\left(\left(2 d_{1}, y_{1}-2\right)\left(0, y_{1}-2+d_{1}\right)\right)$. Note that $C$ is super suitable.

If $d_{1}=4$.

The only remaining graph is $G=G_{54}(4,9)$.

Let $N=\{(1,2),(2,1),(5,0),(5,-2),(6,-1),(6,0),(8,-2)$,$\} .$

1. For a hamiltonian cycle and cycles which miss exactly one vertex from $V(G) \backslash N$ we use a previous case.

2. Cycles missing exactly one vertex from $N$ are presented on Fig. 7. Note that every cycle on Fig. 7 is super suitable. 

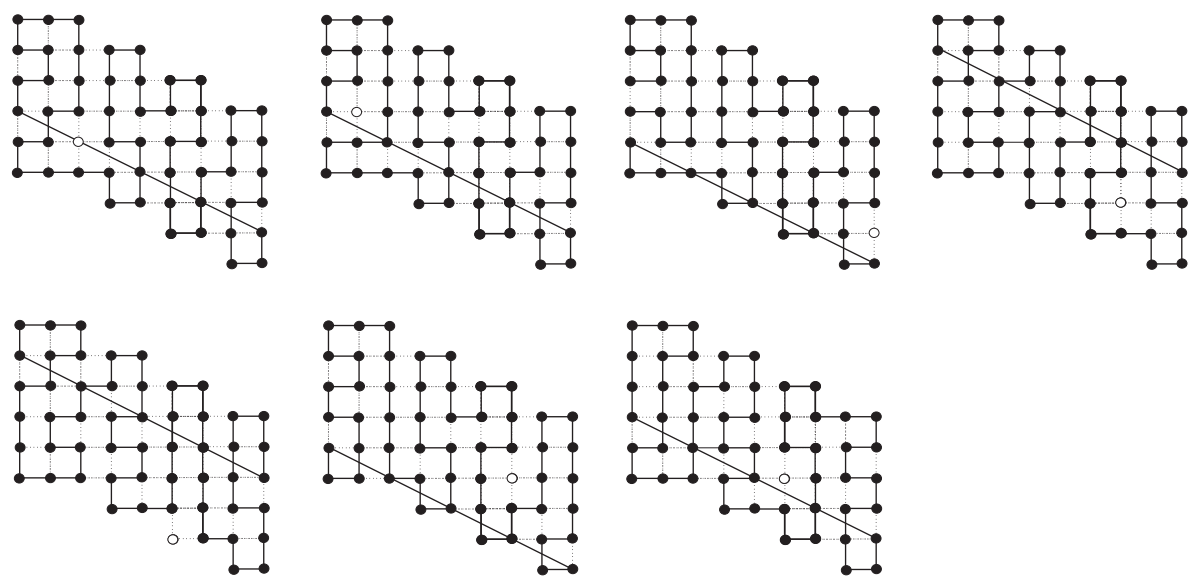

Fig. 7: Near-hamiltonian cycles in $G_{54}(4,9)$

Lemma 10. Let $G=G_{n}\left(d_{1}, d_{2}\right)$ be a connected distance graph with $n=6 d_{2}$, let $B_{i}, 1 \leq i \leq d_{1}-1$, be a 3-block of $G$ and let $(x, y)$ be the vertex of the first column of $B_{i}$. There is a path $P_{E}^{1}$ between vertices $(x, y)$ and $(x+2, y-1)$ containing all vertices of $B_{i}$.

For the proof see Fig. 8 .

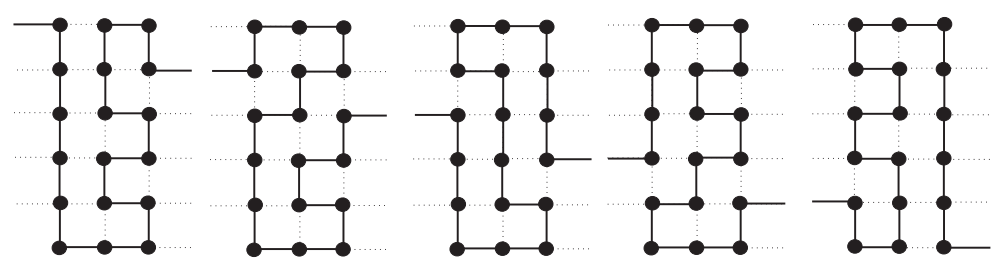

Fig. 8: Paths $P_{E}^{1}$

Lemma 11. Let $G=G_{n}\left(d_{1}, d_{2}\right)$ be a connected distance graph with $n=6 d_{2}$, let $B_{i}, 1 \leq i \leq d_{1}-1$, be a 3-block of $G$ and let $(x, y)$ be the vertex of the first column of $B_{i}$. There are two disjoint paths $S_{1}$ (between $(x, y)$ and $(x+2, y-1)$ ) and $S_{2}$ (between $(x, y+1)$ and $\left.(x+2, y)\right)$ containing (in union) all vertices of $B_{i}$.

For the proof see Fig. 9. 

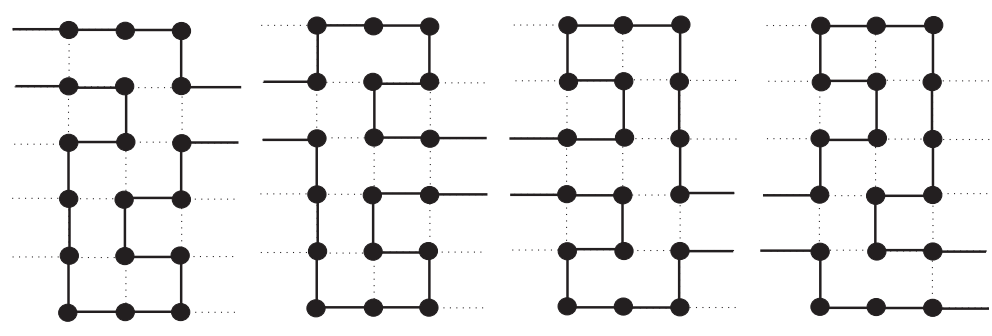

Fig. 9: Paths $S_{1}, S_{2}$

In the sequel, we denote the subgraph induced by edges of $S_{1}$ and $S_{2}$ as $P_{E}^{2}$.

Lemma 12. Let $G \in \mathcal{H}$ be a graph on $n$ vertices consisting of only $k$ 2-blocks and $l 3$-blocks with $k, l \geq 1, k$ even and $n=6(2 k+3 l)$. Then $G$ is hamiltonian and $h(G) \geq n-4(k+l)$.

Proof. Let $\left(w_{1}, w_{2}, \ldots, w_{k+l}\right), w_{j} \in\{2,3\}, j=1,2, \ldots, k+l$, be the characteristic of $G$. If $G$ contains only one 3-block, the statements is true by Lemma 9 . Let $w_{i}=3$. Let $G^{\prime}$ be the graph on $n^{\prime}=n-18$ vertices consisting of blocks $B_{1}, \ldots, B_{i-1}, B_{i+1}, \ldots, B_{k+l}$ and with characteristic $\left(w_{1}, w_{2}, . ., w_{i-1}, w_{i+1}, \ldots, w_{k+l}\right)$, and let $G^{\prime \prime}$ be the graph on $n^{\prime}=n-18$ consisting of blocks $B_{2}, B_{3}, \ldots B_{k+l}$ and with characteristic $\left(w_{2}, w_{3}, \ldots, w_{k+l}\right)$.

If $G$ consists of more that three blocks.

1. A hamiltonian cycle $C$ of $G$ (a cycle $C$ of $G$ which misses exactly one vertex $(x, y)$ with $\left.x \in B_{j}, j \leq i-1\right)$ will be constructed from a hamiltonian cycle $C^{\prime}$ in $G^{\prime}$ (a cycle $C^{\prime}$ of $G^{\prime}$ which misses the vertex $(x, y)$ ) and a path system $P_{E}^{2}$ (a path $P_{E}^{1}$ ) in the block $B_{i}$. The cycle $C$ consists of the following edges:

$\left(\left(x_{1}, y_{1}\right),\left(x_{2}, y_{2}\right)\right)$ if $\left(\left(x_{1}, y_{1}\right),\left(x_{2}, y_{2}\right)\right) \in E\left(C^{\prime}\right)$ and $x_{1} \in B_{j}$ or $x_{2} \in B_{j}$ for $j \leq i-1,\left(\left(x_{1}+3, y_{1}-1\right),\left(x_{2}+3, y_{2}-1\right)\right)$ if $\left(\left(x_{1}, y_{1}\right),\left(x_{2}, y_{2}\right)\right) \in E\left(C^{\prime}\right)$ and $x_{1} \in B_{j}$ or $x_{2} \in B_{j}$ for $j \geq i+1$ and edges of a path $P_{E}^{2}\left(P_{E}^{1}\right)$ in the block $B_{i}$. Note that $C$ is suitable.

2. A cycle $C$ of $G$ which misses exactly one vertex $(x, y)$ with $x \in B_{j}$, $j \geq i+1$ will be constructed from a cycle $C^{\prime}$ in $G^{\prime}$ which misses the vertex $(x-3, y-1)$ and a path $P_{E}^{1}$. The cycle $C$ consists of the following edges:

$\left(\left(x_{1}, y_{1}\right),\left(x_{2}, y_{2}\right)\right)$ if $\left(\left(x_{1}, y_{1}\right),\left(x_{2}, y_{2}\right)\right) \in E\left(C^{\prime}\right)$ and $x_{1} \in B_{j}$ or $x_{2} \in B_{j}$ for $j \leq i-1,\left(\left(x_{1}+3, y_{1}-1\right),\left(x_{2}+3, y_{2}-1\right)\right)$ if $\left(\left(x_{1}, y_{1}\right),\left(x_{2}, y_{2}\right)\right) \in E\left(C^{\prime}\right)$ and $x_{1} \in B_{j}$ or $x_{2} \in B_{j}$ for $j \geq i+1$ and edges of a path $P_{E}^{1}$ in the block $B_{i}$. Note that $C$ is suitable. 
3. A cycle $C$ of $G$ which misses exactly one vertex $(x, y)$ with $x \in B_{i}$ will be constructed from a cycle $C^{\prime \prime}$ in $G^{\prime \prime}$ which misses the vertex $(x-3, y+1)$ and a path $P_{E}^{1}$ in first block. The cycle $C$ consists of the following edges: $\left(\left(x_{1}+3, y_{1}-1\right),\left(x_{2}+3, y_{2}-1\right)\right)$ if $\left(\left(x_{1}, y_{1}\right),\left(x_{2}, y_{2}\right)\right) \in E\left(C^{\prime \prime}\right)$ and edges of a path $P_{E}^{1}$ in the block $B_{1}$. Note that $C$ is suitable.

If $G$ consists of three blocks.

The only remaining graph is $G=G_{48}(3,8)$. Let $N=\{(1,2),(2,1)\}$.

1. For a hamiltonian cycle and cycles which miss exactly one vertex from $V(G) \backslash N$ we use a previous case.

2. Cycles missing exactly one vertex from $N$ are presented on Fig. 10. Note that these cycles are suitable.
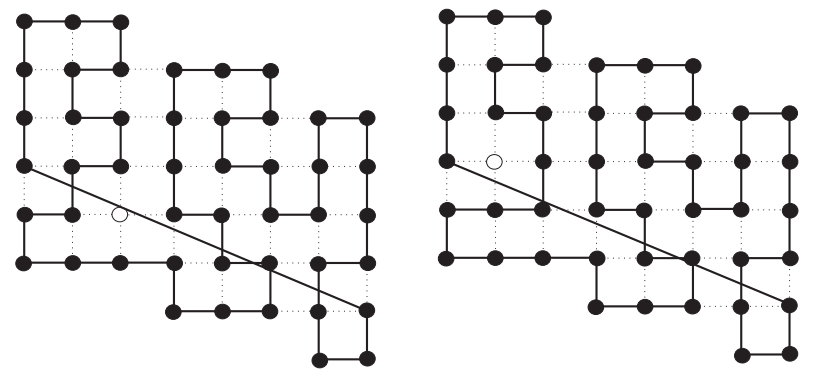

Fig. 10: Near-hamiltonian cycles in $G_{48}(3,8)$

By Lemma 2 and Lemma 12 we obtain:

Corollary 13. Let $G=G_{n}\left(d_{1}, d_{2}\right)$ be a connected non-bipartite distance graph with $2 d_{1}+1 \leq d_{2} \leq 3 d_{1}-1$ and $n=6 d_{2}$. Then $G$ is hamiltonian and $h(G)=n-4 d_{1}$.

Lemma 14. Let $G^{\prime} \in \mathcal{H}$ be a graph on $n^{\prime}$ vertices with characteristic $\left(w_{1}, w_{2}, \ldots, w_{k}\right)$ such that $n=6 \sum_{i=1}^{k} w_{i}$. If $G^{\prime}$ is hamiltonian and $h\left(G^{\prime}\right) \geq n^{\prime}-4 k$, then the graph $G \in \mathcal{H}$ on $n=n^{\prime}+12$ with characteristic $\left(w_{1}, w_{2}, \ldots, w_{i}+\right.$ $\left.2, \ldots, w_{k}\right)$ is hamiltonian as well and $h(G) \geq n-4 k$.

Proof. Let $\left(w_{1}, w_{2}, \ldots, w_{k}\right)$ be the characteristic of $G$ on $n$ vertices. Let $w_{i} \geq 4$, $1 \leq i \leq k$, and let $\left(w_{1}, w_{2}, \ldots, w_{i}-2, \ldots, w_{k}\right)$ be the characteristic of $G^{\prime}$ on $n^{\prime}=n-12$ vertices. The statement is true for every graph consisting of only 2 - and 3 - blocks (by Lemma 12.) and for the graph $G_{24}(1,4)$ (by Lemma 5). 
1. A hamiltonian cycle $C$ of $G$ (a cycle $C$ of $G$ which misses exactly one vertex $(x, y)$, where $\left.x \in B_{j}, j \geq i+1\right)$ will be constructed from a hamiltonian cycle $C^{\prime}$ in $G^{\prime}$ (a cycle $C^{\prime}$ in $G^{\prime}$ which misses the vertex $(x-2, y)$ ). The cycle $C$ consists of the following edges: $\left(\left(x_{1}, y_{1}\right),\left(x_{2}, y_{2}\right)\right)$ if $\left(\left(x_{1}, y_{1}\right),\left(x_{2}, y_{2}\right)\right) \in E\left(C^{\prime}\right)$ and $x_{1} \in B_{j}$ or $x_{2}<B_{j}$ for $j \geq i+1,\left(\left(x_{1}+2, y_{1}\right),\left(x_{2}+2, y_{2}\right)\right)$ if $\left(\left(x_{1}, y_{1}\right),\left(x_{2}, y_{2}\right)\right) \in E\left(C^{\prime}\right)$ and $x_{1} \in B_{i}$ or $x_{2} \in B_{i}$ for $j \leq i-1$. Finally, we replace two biline edge (edges $\left(\left(x_{1}, y_{1}\right),\left(x_{2}, y_{2}\right)\right)$ between two consecutive blocks and $\left(\left(x_{1}, y_{3}\right),\left(x_{1}, y_{4}\right)\right)$ ) by disjoint paths $P_{1}, P_{2}$, respectively, so that the internal vertices of $P_{1}$ and $P_{2}$ (in common) are exactly the new vertices.

2. A cycle $C$ of $G$ which misses exactly one vertex $(x, y)$, where $x \in B_{j}$, $j \leq i-1$ will be constructed from a cycle $C^{\prime}$ in $G^{\prime}$, which misses the vertex $(x, y)$. The cycle $C$ consists of the following edges:

$\left(\left(x_{1}, y_{1}\right),\left(x_{2}, y_{2}\right)\right)$ if $\left(\left(x_{1}, y_{1}\right),\left(x_{2}, y_{2}\right)\right) \in E\left(C^{\prime}\right)$ and $x_{1} \in B_{j}$ or $x_{2} \in B_{j}$, $j \leq i\left(\left(x_{1}+2, y_{1}\right),\left(x_{2}+2, y_{2}\right)\right)$ if $\left(\left(x_{1}, y_{1}\right),\left(x_{2}, y_{2}\right)\right) \in E\left(C^{\prime}\right)$ and $x_{1} \in B_{j}$ or $x_{2} \in B_{j}$ for $j \geq i$. Finally, we replace edges $\left(\left(x_{1}, y_{1}\right),\left(x_{2}, y_{2}\right)\right)$ between two consecutive blocks and $\left(\left(x_{1}, y_{3}\right),\left(x_{1}, y_{4}\right)\right)$ by disjoint paths $P_{1}, P_{2}$, respectively, so that the internal vertices of $P_{1}$ and $P_{2}$ (in common) are exactly the new vertices.

By Lemma 2 and Lemma 14 we obtain:

Corollary 15. Let $G=G_{n}\left(d_{1}, d_{2}\right)$ be a connected non-bipartite distance graph with $\frac{d_{2}}{d_{1}}>2$ and $n=6 d_{2}$. Then $G$ is hamiltonian and $h(G)=n-4 d_{1}$.

Lemma 16. Let $G=G_{n}\left(d_{1}, d_{2}\right)$ be a connected non-bipartite distance graph with $\frac{d_{2}}{d_{1}}>2$ and $n=2 k d_{2}, k \geq 3$. Then $G$ is hamiltonian and $h(G)=$ $n-4 d_{1}$.

Proof. Let $G=G_{n}\left(d_{1}, d_{2}\right)$ be a graph on $n=2 k d_{2}, k \geq 3$, vertices. By Lemma 2 we have $h(G) \geq n-4 d_{1}$. The statement is true for every connected non-bipartite distance graph with $\frac{d_{2}}{d_{1}}>2$ and $n=6 d_{2}$ by corollary 15 . Let $G^{\prime}=G_{n-2 d_{2}}\left(d_{1}, d_{2}\right)$.

A hamiltonian cycle $C$ of $G$ (a cycle $C$ of $G$ which misses exactly one vertex $(x, y)$ ) is obtained from a hamiltonian cycle $C^{\prime}$ of $G^{\prime}$ (a cycle $C^{\prime}$ of $G^{\prime}$ which misses exactly one vertex $(x, y))$. Let $\left(\left(x_{1}, y_{1}\right),\left(x_{2}, y_{2}\right)\right)$ be an edge in $C^{\prime}$, we include into $C$ an edge $\left(\left(x_{1}, y_{1}\right),\left(x_{2}, y_{2}\right)\right)$. Finally, every edge $\left(\left(x_{i}, u\left(x_{i}\right)-\right.\right.$ $\left.2),\left(x_{i}+1, u\left(x_{i}\right)-2\right)\right)$ of $C$, where $x_{i}$ is a column containing 2-vertex is replaced by the path $\left(x_{i}, u\left(x_{i}\right)-2\right),\left(x_{i}, u\left(x_{i}\right)-1,\left(x_{i}, u\left(x_{i}\right)\right),\left(x_{i}+1, u\left(x_{i}\right)\right),\left(x_{i}+2, u\left(x_{i}\right)\right)\right.$, $\ldots,\left(x_{i+1}-1, u\left(x_{i}\right)\right),\left(x_{i+1}-1, u\left(x_{i}\right)-1\right),\left(x_{i+1}-1, u\left(x_{i}\right)-2\right),\left(x_{i+1}-2, u\left(x_{i}\right)-2\right)$, $\ldots,\left(x_{i}+1, u\left(x_{i}\right)-2\right)$. 
Lemma 17. Let $G=G_{n}\left(d_{1}, d_{2}\right)$ be a connected distance graph with $\frac{d_{2}}{d_{1}}>2$ and $n=2 k d_{2}+l\left(d_{1}+d_{2}\right), k \geq 3, l \geq 0$. Then $G$ is hamiltonian and $h(G)=n-4 d_{1}$.

Proof. Let $G=G_{n}\left(d_{1}, d_{2}\right)$ be a distance graph on $n=2 k d_{2}+l\left(d_{1}+d_{2}\right)$ vertices, where $k \geq 3, l \geq 0$. By Lemma 2 we have $h(G) \geq n-4 d_{1}$. The statement is true for every connected non-bipartite distance graph with $\frac{d_{2}}{d_{1}}>2$ and $n=2 k d_{2}$, $k \geq 3$ by Lemma 16 . Let $G^{\prime}=G_{n-d_{1}-d_{2}}\left(d_{1}, d_{2}\right)$.

Let $C_{1}$ be a hamiltonian cycle (a cycle which misses exactly one vertex $(x, y)$ ) of $G$ and $C_{2}$ be a special cycle with $V\left(C_{2}\right)=\left[n+1, n+d_{1}+d_{2}\right]$. Then $C_{1}$ contains an edge $(x, y)=\left(\left(x_{1}, u\left(x_{1}\right),\left(x_{1}+1, u\left(x_{1}\right)\right)\right.\right.$ and $C_{2}$ has an edge $\left(x+d_{2}, y+d_{2}\right)$. Now the set $\bigcup_{\left(x^{\prime}, y^{\prime}\right) \in E\left(C_{1}\right)}\left(x^{\prime}, y^{\prime}\right) \cup \bigcup_{\left(x^{\prime \prime}, y^{\prime \prime}\right) \in E\left(C_{2}\right)}\left(x^{\prime \prime}, y^{\prime \prime}\right)-(x, y)-\left(x+d_{2}, y+d_{2}\right)+$ $\left(x, x+d_{2}\right)+\left(y, y+d_{2}\right)$ is the edge set of the hamiltonian cycle (a cycle which misses exactly one vertex $(x, y))$ of $G^{\prime}$.

Theorem 18. Let $G=G_{n}\left(d_{1}, d_{2}\right)$ be a connected non-bipartite distance graph with $\frac{d_{2}}{d_{1}}>2$ and sufficiently large $n$. Then $G$ is hamiltonian and $h(G)=$ $n-4 d_{1}$.

Proof. By Lemma 17 we have that every connected distance graph with $\frac{d_{2}}{d_{1}}>2$ and $n=2 k d_{2}+l\left(d_{1}+d_{2}\right), k \geq 3, l \geq 0$ is hamiltonian and $h(G)=n-4 d_{1}$. Since $2 d_{2}$ and $d_{1}+d_{2}$ are comprime, it follows (from Sylvester formula for closed form of the solution of the Frobenius coin problem) that every sufficiently large integer (more precisely, larger than $6 d_{2}\left(d_{1}+d_{2}-1\right)\left(2 d_{2}-1\right)$ ) is a positive integer linear combination of $2 d_{2}$ and $d_{1}+d_{2}$.

\section{Lower Bound for Bipartite Graphs}

In this section, we establish lower bound of $\mathrm{H}$-force number for bipartite graphs satisfying $\frac{d_{2}}{d_{1}}>2$.

Lemma 19. The graph $G=G_{18}(1,3)$ is hamiltonian and $h(G)=8$.

Proof. It is easy to check that $G$ is hamiltonian and there is a hamiltonian cycle with one biline pair of edges do not belonging to the only block of $G$ (see first cycle on Fig.11). By the Lemma 3 we have $h(G) \leq 8$. Moreover, there are 8 cycles in $G$ missing exactly one pair of different vertices, by claim 4 at least one vertex of a pair belongs to any $\mathrm{H}$-force set (due to automorphism of $G$ mapping any vertex $(x, y)$ to the vertex $n-(x, y)-1$ we present only 4 of mentioned cycles on Figure 11). Note that all these cycles are suitable. 

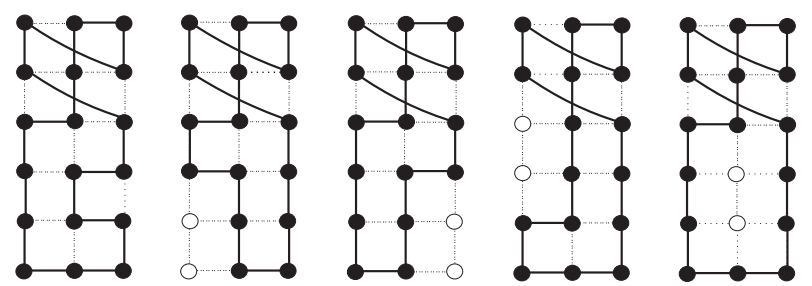

Fig. 11: Hamiltonian cycle and near-hamiltonian cycles in $G_{18}(1,3)$

Lemma 20. Let $G=G_{n}\left(d_{1}, d_{2}\right)$ be a connected distance graph with odd $d_{1}, d_{2}=2 d_{1}+1$ and $n=6 d_{2}$. Then $G$ is hamiltonian and $h(G)=\frac{n}{2}-d_{1}$.

Proof. Let $G=G_{6\left(2 d_{1}+1\right)}\left(d_{1}, 2 d_{1}+1\right)$ be a distance graph with odd $d_{1}$. The characteristic of $G$ is $(3,2, \ldots, 2)$, with even number of 2-blocks. By Lemma 3, we have $h(G) \leq \frac{n}{2}-d_{1}$; moreover, this bound is sharp for the graph $G_{18}(1,3)$. Let $G^{\prime}=G_{6\left(2 d_{1}-3\right)}\left(d_{1}-2,2 d_{1}-3\right)$.

If $d_{1} \geq 5$ see the proof of Lemma 9 , part $d_{1} \geq 5$.

If $d_{1}=3$. The only remaining graph is $G=G_{42}(3,7)$.

1. For a hamiltonian cycle and cycles which misses exactly two vertices, namely $(0,0)$ and $(0,1)$ or $(2,0)$ and $(2,1)$ or $(0,2)$ and $(0,3)$ or $(1,1)$ and $(1,2)$ we use previous case.

2. Cycle which misses exactly two vertices, namely $(3,-1)$ and $(3,0)$ or $(5,-2)$ and $(5,-1)$ or $(6,-1)$ and $(6,0)$ or $(4,0)$ and $(4,1)$ or $(3,1)$ with $(5,0)$ are presented on Fig. 12. Note that all cycles on Fig. 12 are super suitable.
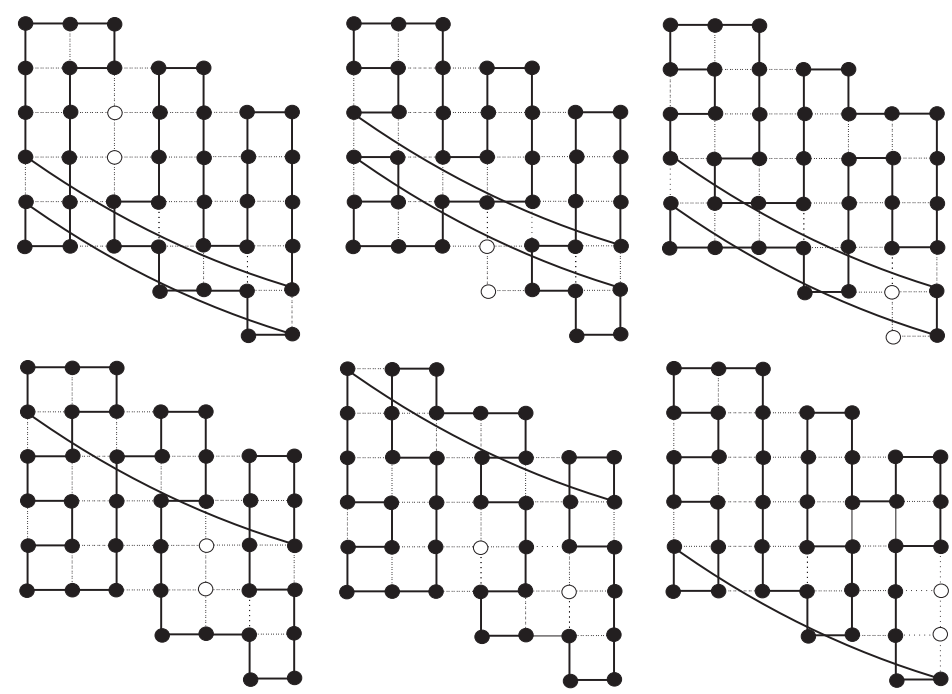
Fig. 12: Near-hamiltonian cycles in $G_{42}(3,7)$

Lemma 21. Let $G \in \mathcal{H}$ be a graph on $n$ vertices consisting of only $k$ 2-blocks and $l$ 3-blocks with $k, l \geq 1, k$ odd and $n=6(2 k+3 l)$. Then $G$ is hamiltonian and $h(G) \geq n-4(k+l)$.

The proof goes in analogous way as the proof of Lemma 12 .

By Lemma 3 and Lemma 21 we obtain:

Corollary 22. Let $G=G_{n}\left(d_{1}, d_{2}\right)$ be a connected bipartite distance graph with $2 d_{1}+1 \leq d_{2} \leq 3 d_{1}-1$ and $n=6 d_{2}$. Then $G$ is hamiltonian and $h(G)=\frac{n}{2}-d_{1}$.

Lemma 23. Let $G^{\prime} \in \mathcal{H}$ be a graph on $n^{\prime}$ vertices with characteristic $\left(w_{1}, w_{2}, \ldots, w_{k}\right)$ such that $n=6 \sum_{i=1}^{k} w_{i}$. If $G^{\prime}$ is hamiltonian and $h\left(G^{\prime}\right) \geq \frac{n}{2}-d_{1}$, then the graph $G \in \mathcal{H}$ on $n=n^{\prime}+12$ with characteristic $\left(w_{1}, w_{2}, \ldots, w_{i}+\right.$ $\left.2, \ldots, w_{k}\right)$ is hamiltonian as well and $h(G) \geq \frac{n}{2}-d_{1}$.

The proof goes in analogous way as the proof of Lemma 15 .

By Lemma 3 and Lemma 23 we obtain:

Corollary 24. Let $G=G_{n}\left(d_{1}, d_{2}\right)$ be a connected bipartite distance graph with $\frac{d_{2}}{d_{1}}>2$ and $n=6 d_{2}$. Then $G$ is hamiltonian and $h(G)=\frac{n}{2}-d_{1}$.

In analogous way as in proofs of lemmas of the final part of previous Section (details are left to the reader), we can show

Lemma 25. Let $G=G_{n}\left(d_{1}, d_{2}\right)$ be a connected bipartite distance graph with $\frac{d_{2}}{d_{1}}>2$ and $n=2 k d_{2}, k \geq 3$. Then $G$ is hamiltonian and $h(G)=\frac{n}{2}-d_{1}$.

Lemma 26. Let $G=G_{n}\left(d_{1}, d_{2}\right)$ be a connected bipartite distance graph $\frac{d_{2}}{d_{1}}>2$ and $n=2 k d_{2}+l\left(d_{1}+d_{2}\right), k \geq 3, l \geq 0$. Then $G$ is hamiltonian and $h(G)=\frac{n}{2}-d_{1}$.

Theorem 27. Let $G=G_{n}\left(d_{1}, d_{2}\right)$ be a connected bipartite distance graph with $\frac{d_{2}}{d_{1}}>2$ and with sufficiently large $n$. Then $G$ is hamiltonian and $h(G)=\frac{n}{2}-d_{1}$.

\section{Acknowledgments}

Supported in part by Slovak VEGA grant 1/0652/12. sidd 


\section{References}

[1] G. Chartrand, S.F. Kapoor, D.R. Lick: n-hamiltonian graphs, J. Combin. Theory, 9, No. 3 (1970), 308-312, doi: 10.1016/S0021-9800(70)80069-2

[2] V. Chvátal, P. Erdös, A note on hamiltonian circuits, Discrete Math., 2, No. 2 (1972), 111-113, doi: 10.1016/0012-365X(72)90079-9

[3] R. Diestel, Graph theory, Springer, 2000.

[4] I. Fabrici, E. Hexel, S. Jendrol', On vertices enforcing a hamiltonian cycle, Discuss. Math. Graphs Theory, 33, No. 1 (2013), 71-89, doi: 10.7151/dmgt.1653

[5] F. Göring, J. Harant, Hamiltonian cycles through prescribed edges of at least 4-connected maximal planar graphs, Discrete Math., 310, No. 9 (2010), 1491-1494, doi: 10.1016/j.disc.2009.10.005

[6] E. Grinberg, Plane homogeneous graphs of degree 3 without hamiltonian circuits, Latvian Math. Yearbook, (1968) 51-58 (in Russian).

[7] J. Harant, S. Senitsch: A generalization of Tutte's Theorem on hamiltonian cycles in planar graphs, Discrete Math., 309, No. 15 (2009), 4949-4951, doi: $10.1016 /$ j.disc.2008.04.038

[8] Ch. Löwenstein, D. Rautenbach, F. Regen, On Hamiltonian paths in distance graphs, Appl. Math. Letters, 24, No. 7 (2011), 1075-1079, doi: 10.1016/j.aml.2011.01.025

[9] Ch. Löwenstein, D. Rautenbach, R. Soták, On hamiltonian paths and cycles in sufficiently large distance graphs, Discrete Math. Theor. Comput. Sci., 16 (2014), 7-30.

[10] D.A. Nelson, Hamiltonian Graphs, Master thesis, Vanderbilt University, 1973.

[11] D.P. Sanders, On paths in planar graphs, J. Graph Theory, 24, No. 4 (1997), 341-345, doi: 10.1002/(SICI)1097-0118(199704)24:4<3C3 41::AIDJGT6>3E3.0.CO;2-O

[12] M. Timková, Enforced hamiltonian cycles in generalized dodecahedra, Electron. J. Graph Theory Appl., 1, No. 2 (2013), 77-88, doi: 10.5614/ejgta.2013.1.2.1 
